\title{
FAUNA DE COLEÓPTEROS LAMELICORNIOS DE SANTIAGO XIACUI, SIERRA NORTE, OAXACA, MÉXICO
}

\author{
Andrés Ramírez-Ponce, Jazive Allende-Canseco y Miguel Angel Morón \\ Departamento de Biología de Suelos, Instituto de Ecología, A. C. Apdo. Postal 63, Xalapa, Veracruz \\ 91000, MÉXICO. e-mail: miguel.moron@inecol.edu.mx
}

Ramírez-Ponce, A., J. Allende-Canseco y M. A. Morón. 2009. Fauna de coleópteros lamelicornios de Santiago Xiacui, Sierra Norte, Oaxaca, México. Acta Zoológica Mexicana (n. s.), 25(2): 323-343.

RESUMEN. Se presenta un estudio comparativo de la fauna de coleópteros lamelicornios (Melolonthidae, Scarabaeidae y Passalidae) establecida en el bosque mesófilo de montaña, y los bosques de pinos y encinos situados entre los 1,946 y 2,363 m de altitud en el municipio de Santiago Xiacui, Sierra Norte del estado de Oaxaca, México. Entre junio de 2003 y junio de 2004 se colectaron 4,317 ejemplares que representan a 74 especies de 36 géneros incluídos en 11 subfamilias y 23 tribus. Onthophagus chevrolati retusus Harold fue la especie mas abundante, equivalente al $67.7 \%$ de la muestra total. Otras especies abundantes fueron Deltochilum mexicanum Burmeister, Phyllophaga vetula (Horn), P. ravida (Blanchard), Paranomala inconstans (Burmeister), Xyloryctes telephus y Vindex agnoscendus Percheron. Los adultos de la mayoría de las especies están activos entre junio y agosto. Se destaca la importancia de las especies de Phyllophaga, Paranomala, Xyloryctes, Deltochilum, Onthophagus y Vindex, como consumidoras de raíces, follaje, hojarasca, madera muerta, excrementos y carroña. Se citan cinco especies de Phyllophaga, Paranomala, Peltotrichius y Copris previamente no registradas en Oaxaca.

Palabras clave. Scarabaeoidea, taxonomía, distribución, bosque mesófilo de montaña, pinares, encinares.

Ramírez-Ponce, A., J. Allende-Canseco \& M. A. Morón. 2009. The scarab beetles of Santiago Xiacui, Sierra Norte, Oaxaca, Mexico. Acta Zoológica Mexicana (n. s.), 25(2): 323-343.

ABSTRACT. A comparative study of the species richness, abundance and phenology of the Scarabaeoidea beetle fauna of the mountains of Santiago Xiacui, Oaxaca, Mexico is presented. Monthly data were obtained during June 2003 to June 2004 in pine-oak forests and cloud florests, located at 1,946-2,363 m of altitude. Records of 74 species, 36 genera, 23 tribes and 11 subfamilies of Melolonthidae, Scarabaeidae and Passalidae, represented by 4,317 specimens, are included. Onthophagus chevrolati retusus Harold, Deltochilum mexicanum Burmeister, Phyllophaga vetula (Horn), P. ravida (Blanchard), Paranomala inconstans (Burmeister), Xyloryctes telephus and Vindex agnoscendus Percheron, were the most abundant species in the samples. Adults of many species are active during May to August. High species richness was found in the forests, where the species of Phyllophaga, Paranomala, Xyloryctes, Deltochilum, Onthophagus and Vindex, are important consumers of roots, leaves, leaflitter, rotten wood, dung and carrion. Five species of Phyllophaga, Paranomala, Peltotrichius, and Copris are recorded by the first time for the state of Oaxaca.

Key Words: Scarabaeoidea, taxonomy, distribution, cloud forest, pine-oak forest.

Recibido: 1/07/2008; aceptado: 5 /02/2009. 


\section{INTRODUCCIÓN}

La gran diversidad y abundancia de las especies de coleópteros lamelicornios tienen repercusiones ecológicas y económicas importantes, debido principalmente a la amplia variedad de hábitos alimenticios y preferencias bióticas que presentan (Morón 1985, 1997, 2001, Castillo y Morón 1992, Morón et al. 1996, Morón y Aragón, 2003). La fauna de escarabajos refleja algunos de los cambios antropogénicos en un ecosistema, por lo que se ha propuesto usarlos como indicadores biológicos o parámetros ecológicos (Morón y Terrón 1984, Halffter et al. 1992, Favila y Halffter 1997, Morón 1997) ya que permiten estimar la importancia de una zona para considerarla como área natural protegida (Morón 2003a).

A pesar de que muchas regiones del estado de Oaxaca han sido visitadas con frecuencia por naturalistas, entomólogos y colectores desde mediados del siglo XIX, a la fecha no se dispone de un inventario estatal de los coleópteros lamelicornios, ni de estudios faunísticos regionales o locales, comparables a los efectuados con lepidópteros (Luis-Martínez et al. 1991). En una aproximación preliminar Morón (2003a) ha citado que la riqueza de lamelicornios de Oaxaca alcanza las 389 especies, cifra que sitúa al estado en el tercer puesto dentro del país, después de Chiapas (455 especies) y Veracruz (430 especies).

Como parte del proyecto a largo plazo para evaluar y comparar la fauna de Coleoptera Lamellicornia o Scarabaeoidea en los distintos ambientes característicos de la Zona de Transición Mexicana iniciado por M. A. Morón en 1974, se planeó una prospección en los alrededores de Santiago Xiacui, Sierra Norte de Oaxaca, tomando en cuenta que dicha zona esta incluída entre las regiones terrestres prioritarias para su conservación en México (CONABIO-RPT-130, Sierra del Norte Oaxaca-Mixe), según los criterios definidos por la Comisión Nacional para el Conocimiento y Uso de la Biodiversidad (Arriaga et al. 2000).

Los objetivos del presente estudio fueron: a) obtener una lista de las especies de coleópteros lamelicornios de Santiago Xiacui, y estimar su diversidad y, b) analizar los registros obtenidos a lo largo de un año en relación con las variables climáticas, altitudinales y de vegetación.

\section{DESCRIPCIÓN DEL ÁREA DE ESTUDIO}

La localidad pertenece a la provincia biogeográfica de la Sierra Madre del Sur en la región de la Sierra Norte del estado de Oaxaca (Fig. 1), pertenece al distrito de Ixtlán de Juárez y cuenta con una superficie de $67.62 \mathrm{Km}^{2}$. Está situada a una altitud promedio de 2,000 msnm entre las coordenadas $17^{\circ} 17^{\prime} 33^{\prime}$ " de latitud norte y $96^{\circ} 26^{\prime}$ 00" de longitud oeste. Los tipos de suelo predominantes son acrisol húmico, cambisol y litosol vertico, regosol eutrico, luvisol vertico y cambisol húmico. La vegetación comprende asociaciones de pinos y encinos, bosque 
mesófilo de montaña y bosque de galerías. El clima es templado subhúmedo con una temperatura media anual de $17.2^{\circ} \mathrm{C}$ y una precipitación media de $1,183.6 \mathrm{~mm}$ anuales (INEGI 1998). Es una zona donde la diversidad biológica de México tiende a incrementarse, pues es en el centro-noreste de Oaxaca donde convergen la Sierra Madre del Sur, el Eje Neovolcánico y la Sierra Madre Oriental, aquí es donde se presenta la mayor heterogeneidad de hábitats y la más compleja historia geológica de México (Espinosa et al. 2000). La agricultura solo se practica en las proximidades de la zona urbana entre los 1,900 y 2,300 msnm. Los cultivos con mayor importancia son maíz, frijol, flores ornamentales y frutas como membrillo, durazno, manzana, tejocote, nuez y ciruela. La actividad pecuaria está restringida a pocos rebaños de caprinos, ovinos y escaso ganado vacuno, cuya presencia se limita a las cercanías del poblado.

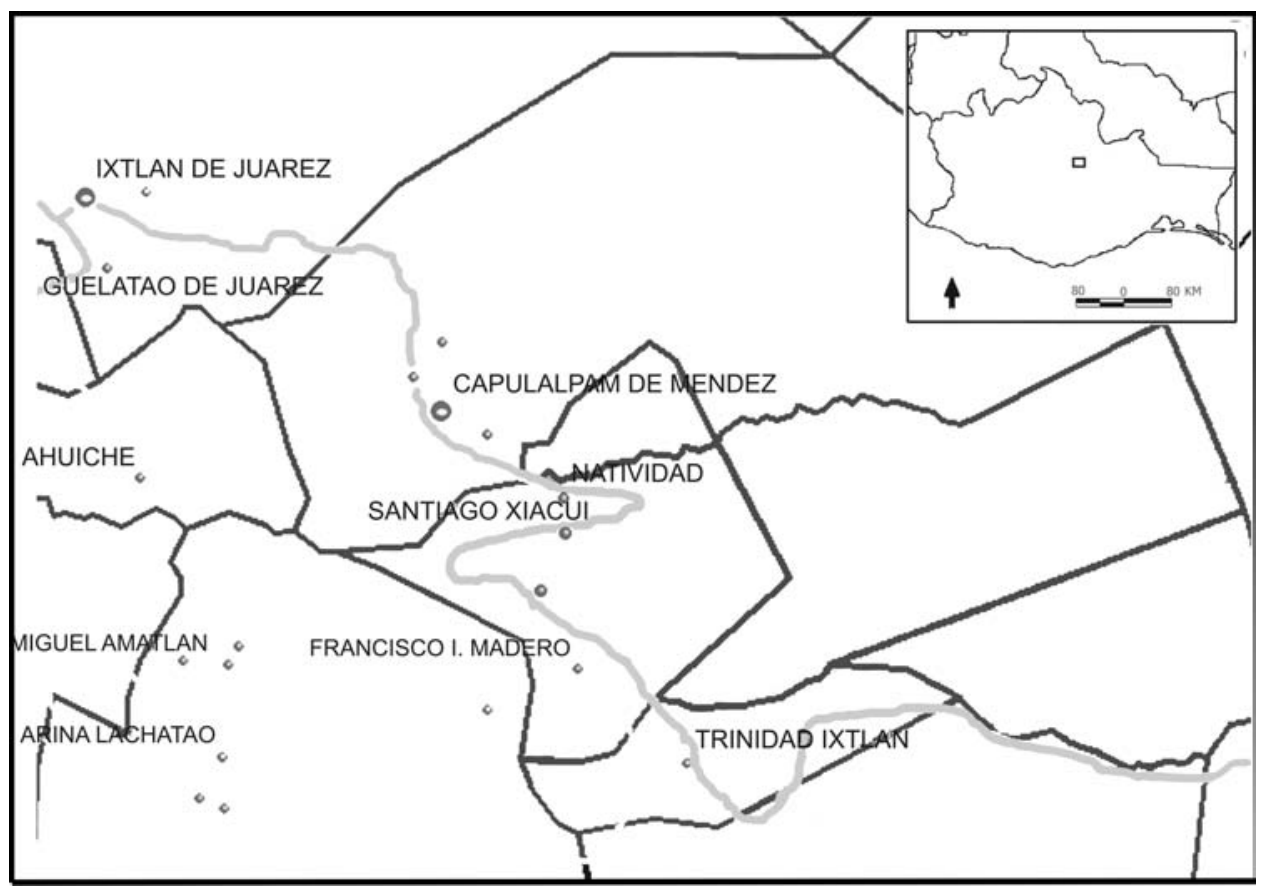

Figura 1. Ubicación del municipio de Santiago Xiacui, Oaxaca (INEGI). 


\section{MATERIAL Y MÉTODOS}

Mediante el reconocimiento del lugar y consultando cartas topográficas y de vegetación, se seleccionaron 11 sitios en los cuales se instalaron trampas. Estos sitios representan los diferentes tipos de vegetación y altitudes de Santiago Xiacui (Cuadro 1). También se utilizó el trayecto entre las trampas (aprox. $3 \mathrm{~km}$ ) para realizar colecta directa de ejemplares. Se efectuaron muestreos sistemáticos de una semana de duración desde junio de 2003 hasta junio de 2004. Mensualmente se instalaron 11 necrotrampas permanentes NTP-80 (Morón y Terrón 1984), seis coprotrampas convencionales, seis carpotrampas con plátano fermentado y una trampa de luz fluorescente tipo pantalla para las colectas nocturnas, además de realizar colecta directa no sistemática bajo el alumbrado público, en troncos y tocones podridos, bajo excremento de ganado y de fauna silvestre, en el suelo, en hojarasca, en flores y follaje. Los ejemplares colectados fueron montarlos en alfiler antes de identificarlos. Se siguió la clasificación supragenérica de Endrödi (1966), Reyes-Castillo (1970, 2003), Morón et al. (1997) y Morón (2003a). Todo el material estudiado está depositado en la colección entomológica del Instituto de Ecología, A.C. Xalapa (IEXA) y en la colección particular M. A. Morón, Xalapa (MXAL).

Cuadro 1. Características de los sitios de colecta.

\begin{tabular}{|c|c|c|c|c|c|}
\hline Sitios & Nombre & Vegetación & $\begin{array}{l}\text { Altitud } \\
\text { (Msnm) }\end{array}$ & $\begin{array}{l}\text { Latitud } \\
\text { Norte }\end{array}$ & $\begin{array}{l}\text { Longitud } \\
\text { Oeste }\end{array}$ \\
\hline 1 & El Aserradero & Bosque de encino & 2148 & $17^{\circ} 17^{\prime} 7.3^{\prime \prime}$ & $96^{\circ} 29^{\prime} 48.6^{\prime \prime}$ \\
\hline 2 & Río a las cabañas & Bosque de galerías & 2072 & $17^{\circ} 17^{\prime} 15.3^{\prime \prime}$ & $96^{\circ} 25^{\prime} 24.8^{\prime \prime}$ \\
\hline 3 & Brecha arrastradero & Bosque de encino-pino & 2306 & $17^{\circ} 17^{\prime} 36.9^{\prime \prime}$ & $96^{\circ} 24^{\prime} 24.8^{\prime \prime}$ \\
\hline 4 & Cañada del Vivero & Bosque mesófilo & 2319 & $17^{\circ} 17^{\prime} 28.5^{\prime \prime}$ & $96^{\circ} 24^{\prime} 16.0^{\prime \prime}$ \\
\hline 5 & El Vivero & Bosque de pino-encino & 3240 & $17^{\circ} 17^{\prime} 25.7^{\prime \prime}$ & $96^{\circ} 24^{\prime} 21.4^{\prime \prime}$ \\
\hline 6 & El Arroyo & Bosque de galerías & 2363 & $17^{\circ} 17^{\prime} 17.4^{\prime \prime}$ & $96^{\circ} 24^{\prime} 10.8^{\prime \prime}$ \\
\hline 7 & El Quelite & Bosque de pino-encino & 2351 & $17^{\circ} 17^{\prime} 33.7^{\prime \prime}$ & $96^{\circ} 23^{\prime} 12.7^{\prime \prime}$ \\
\hline 8 & Punto escondido & Bosque de pino & 2357 & $17^{\circ} 18^{\prime} 2.6^{\prime \prime}$ & $96^{\circ} 23^{\prime} 19.1^{\prime \prime}$ \\
\hline 9 & Puente Cervantes & Bosque de galerías & 2262 & $17^{\circ} 17^{\prime} 27.1^{\prime \prime}$ & $96^{\circ} 22^{\prime} 44.2^{\prime \prime}$ \\
\hline 10 & Bosque Madriguera & Bosque mesófilo & 2272 & $17^{\circ} 18^{\prime} 1.8^{\prime \prime}$ & $96^{\circ} 22^{\prime} 49.3^{\prime \prime}$ \\
\hline 11 & Llano Verde & Bosque de pino-encino & 2265 & $17^{\circ} 18^{\prime} 15.8^{\prime \prime}$ & $96^{\circ} 22^{\prime} 21.4^{\prime \prime}$ \\
\hline 12 & Santiago Xiacui & $\begin{array}{l}\text { Bosque de pino y } \\
\text { vegetación inducida }\end{array}$ & 2027 & $17^{\circ} 17^{\prime} 33.6^{\prime \prime}$ & $96^{\circ} 26^{\prime} 5.6^{\prime \prime}$ \\
\hline 13 & San Pedro Nolasco & $\begin{array}{l}\text { Bosque de galerías y } \\
\text { vegetación inducida }\end{array}$ & 1946 & $17^{\circ} 17^{\prime} 44.7^{\prime \prime}$ & $96^{\circ} 25^{\prime} \quad 35.4^{\prime \prime}$ \\
\hline
\end{tabular}


Se realizó la predicción de la riqueza total del lugar considerándolo como el valor del número de especies en el que una curva de acumulación de especies ha alcanzado su asíntota (Soberón y Llorente 1993). Con ayuda de los programas Effort Predictor V. 1.0 y EstimateS V. 7 se hizo una estimación paramétrica del número máximo de especies (Smax) mediante el ajuste de una curva acumulada basándose en el modelo de distribución de abundancias Log-normal (Chao 2004, Moreno 2001) y de la cantidad del esfuerzo de colecta requerida para encontrar un cierto porcentaje del número de especies pronosticadas con un valor de 1,000 combinaciones aleatorias de las diferentes muestras (Moreno y Halffter 2000, Colwell 2004). Además se comparó este resultado con otros estimadores no paramétricos que solo necesitan datos de presencia-ausencia (Chao 2, Jacknife de primer orden, Jacknife de segundo orden y Bootstrap) (Moreno 2001, Espinosa 2003, Colwell 2004).

\section{RESULTADOS}

Composición faunística. Durante el año de colecta se capturaron 4,317 ejemplares de Melolonthidae, Scarabaeidae y Passalidae, incluidos en 11 subfamilias, 23 tribus, 36 géneros y 74 especies (Cuadros 2-3).

\section{FAMILIA MELOLONTHIDAE.}

Phyllophaga Harris, 1826. Fue posible capturar 319 ejemplares de 16 especies (Cuadro 2), tres de ellas corresponden a especies no descritas de los grupos "ravida", "scissa" y a un grupo nuevo del subgénero Phytalus. Las especies mas abundantes fueron: $P$. vetula y $P$. ravida. Ambas especies tienen amplia distribución en México y sus larvas se han citado como plagas subterráneas de varios cultivos básicos (Morón 2003b). Las especies consideradas como raras o con distribución restringida en Oaxaca y Chiapas son: P. (Phytalus) lulaana Morón, P. (Phytalus) lineatoides Morón, $P$. (Phytalus) tejupana Morón, P. (Phyllophaga) punctulicollis (Bates) y $P$. (Phyllophaga) nisuens Saylor.

Diplotaxis Kirby, 1837. Se colectaron 108 ejemplares que representan a cinco especies (Cuadro 2), ninguna de las cuales se identificó con precisión, y es posible que correspondan a taxones no descritos. La morfoespecie más abundante fue Diplotaxis sp. aff. jacala.

Macrodactylus Latreille, 1825. Se colectaron 11 machos y 12 hembras de $M$. mexicanus alimentándose sobre follaje de plantas no identificadas. Fue posible observar una gran actividad de varios cientos de individuos, volando o formando parejas o tríos sobre las hojas.

Isonychus Mannerheim, 1829. Fue posible obtener 13 machos y 12 hembras de $I$. sp. aff. arizonensis atraídos por las luces. Para confirmar la identidad precisa de estos ejemplares es necesario revisar el género al menos para México y América Central. 
Cuadro 2. Abundancia mensual de Melolonthidae en Santiago Xiacui, Oaxaca.

\begin{tabular}{|c|c|c|c|c|c|c|c|c|c|c|c|c|c|c|}
\hline Especies & $\mathbf{J}$ & $\mathbf{J}$ & $\mathbf{A}$ & $\mathbf{S}$ & $\mathbf{O}$ & $\mathbf{N}$ & D & $\mathbf{E}$ & $\mathbf{F}$ & $\mathbf{M}$ & $\mathbf{A}$ & $\mathbf{M}$ & & Total \\
\hline Paranomala ayjikcala (Morón \& Nogueira, 2002) & & & & & & & & & & & & 1 & & 1 \\
\hline P. undulata (Melsheimer, 1844) & & & & & & & & & & & & 66 & 2 & 68 \\
\hline P. inconstans (Burmeister, 1844) & & 1 & 1 & & & & & & & & 4 & 122 & 1 & 129 \\
\hline P. aff. inconstans (Burmeister, 1844) & & & & & & & & & & & & 2 & & 2 \\
\hline$P$. aff. chevrolati (Bates, 1888) & & & & & & & & & & & & 1 & & 1 \\
\hline P. aff. discoidalis (Bates, 1888) & & & & & & & & & & & & 1 & & 1 \\
\hline$P$. aff. denticollis (Bates, 1888) & & & & & & & & & 1 & 1 & 12 & 18 & & 32 \\
\hline Paranomala sp. 1 & & & & & & & & & & & & 26 & 1 & 27 \\
\hline Paranomala sp. 2 & & & & & & & & & & & & 1 & & 1 \\
\hline Chrysina macropus (Francillon, 1795) & 2 & 3 & & 1 & & & & & & & 3 & 8 & 2 & 19 \\
\hline Plusiotis adelaida (Hope, 1840) & 8 & 1 & 4 & & 3 & 1 & & & & & 1 & 1 & & 19 \\
\hline Plusiotis sp. & 3 & & & 1 & & & & & & & & & & 4 \\
\hline Rutelisca flohri Bates, 1888 & & & & & & & & & & & & & 1 & 1 \\
\hline Macrodactylus mexicanus Burmeister, 1845 & & 1 & & & & & & & & & & 1 & 21 & 13 \\
\hline Isonychus arizonensis Howden, 1959 & & & & & & & & & & & & 24 & 1 & 25 \\
\hline Hoplia sp. & & & & & & & & & & & 1 & & & 1 \\
\hline Diplotaxis aff. denticeps Bates, 1887 & & & & & & & & & & & & 7 & & 7 \\
\hline D. aff. jacala Vaurie, 1960 & 1 & & & & & & & & & & 9 & 46 & 4 & 60 \\
\hline D. aff. circulans Vaurie, 1960 & 2 & 3 & 3 & 4 & 6 & 2 & & & & & & 9 & 4 & 33 \\
\hline Diplotaxis sp. 1 & & & & & & & & & & & & & 2 & 2 \\
\hline Diplotaxis sp. 2 & & & 1 & & 1 & & & 3 & 1 & & & & & 6 \\
\hline Phyllophaga lulana Morón, 2000 & 1 & 2 & 1 & & & & & & & & & 1 & 1 & 6 \\
\hline P. lineatoides Morón, 2000 & & & & & & & & & & & & 6 & & 6 \\
\hline P. tejupana Morón, 2000 & & & & & & & & & & & & 1 & & 1 \\
\hline P. obsoleta (Blanchard, 1850) & & & & & & & & & & & & 1 & & 1 \\
\hline P. macrocera (Bates, 1888) & & & & & & & & & & & & 34 & & 34 \\
\hline Phyllophaga sp. 1 & & & & 1 & 1 & & & & & & & 4 & 2 & 8 \\
\hline P. ravida (Blanchard, 1850) & & & & & & & & & & & & 77 & 3 & 80 \\
\hline Phyllophaga sp. 4 & & & & & & & & & & & & 3 & 1 & 4 \\
\hline P. heteronycha (Bates, 1888) & & & & & & & & & & & & 5 & & 5 \\
\hline P. punctullicollis (Bates, 1888) & & & 1 & & & & & & & & & 1 & & 2 \\
\hline P. leonina (Bates, 1888) & & 1 & & & & & & & & & & 1 & & 2 \\
\hline Phyllophaga sp. 2 & & & 2 & 1 & & & & & & & & & & 3 \\
\hline P. vetula (Horn, 1887) & & & 1 & & & & & & & & 9 & 135 & 7 & 152 \\
\hline P. eniba Saylor, 1943 & & 1 & & & & & & & & & & 8 & & 9 \\
\hline P. nisuens Saylor, 1937 & & & & & & & & & & & & & 1 & 1 \\
\hline Phyllophaga sp. 3 & & & & & & & 5 & & & & & & & 5 \\
\hline Ancognatha falsa (Arrow, 1911) & 3 & & & & & & & & & & & 1 & & 4 \\
\hline A. quadripunctata Bates, 1888 & & & & & & & & & & & & & 1 & 1 \\
\hline Dynastes hyllus Chevrolat, 1843 & 1 & & & & & & & & & & & & & 1 \\
\hline Golofa imperialis Thomson, 1858 & 1 & 2 & 2 & & & & & & & & & & & 5 \\
\hline Xyloryctes telephus Burmeister, 1847 & 5 & & & 1 & & & & 1 & & & 3 & & & 10 \\
\hline Heterogomphus chevrolati Burmeister, 1847 & & & & & & & & & & & & 1 & & 1 \\
\hline Orizabus clunalis (LeConte, 1856) & & & 2 & & 1 & & & & & & & & 1 & 4 \\
\hline O. aff. batesi (Prell, 1914) & & & & & & & & & & & & 1 & & 1 \\
\hline Ligyrus sallei (Bates, 1888) & & & & & & & & & & & 1 & & & 1 \\
\hline Euphoria westermanni Gory \& Percheron, 1833 & & & & & & & & & & & & 3 & 1 & 4 \\
\hline Cotinis mutabilis (Gory \& Percheron, 1833) & & & & & & & & & & & & 1 & & 1 \\
\hline Peltotrichius linea (Burmeister, 1841) & & & & & & & & & & & & & 1 & 1 \\
\hline TOTALES & 27 & 15 & 18 & 9 & 12 & 3 & 5 & 4 & 2 & 1 & 43 & 618 & & 3805 \\
\hline
\end{tabular}


Cuadro 3. Abundancia mensual de Scarabaeidae y Passalidae en Santiago Xiacui, Oaxaca.

\begin{tabular}{|c|c|c|c|c|c|c|c|c|c|c|c|c|c|c|}
\hline Especies & $\mathbf{J}$ & $\mathbf{J}$ & A & $\mathbf{S}$ & $\mathbf{O}$ & $\mathbf{N}$ & D & $\mathbf{E}$ & $\mathbf{F}$ & M & $\mathbf{A}$ & $\mathbf{M}$ & $\mathbf{J}$ & Total \\
\hline Geotrupes nebularum Howden, 1964 & & & & 1 & 1 & & 1 & & & & 2 & 3 & & 8 \\
\hline G. sallei Jekel, 1865 & & 1 & 2 & & 2 & 1 & & & & & & 2 & 6 & 14 \\
\hline Ochodaeus aff. luridus Westwood, 1852 & & & & & 1 & & & & & & & & & 1 \\
\hline Labarrus pseudolividus Balthasar, 1941 & & & 1 & & & & & & & & & 1 & 5 & 7 \\
\hline Haroldiellus sallei (Harold, 1863) & & & & & & & & & & & & 12 & & 12 \\
\hline Gonaphodiellus opisthius (Bates, 1887) & & & & & 1 & 171 & 1 & 2 & & 1 & & 19 & 5 & 46 \\
\hline Liothorax aff. innexus (Say, 1835) & & & & & & & & & & & & & 4 & 4 \\
\hline Ataenius aff. cribrithorax Bates, 1887 & & & & & & & & & & & & 5 & & 5 \\
\hline Copris armatus Harold, 1869 & & & & & 1 & 1 & & & & & & 2 & 1 & 5 \\
\hline Dichotomius colonicus Say, 1835 & & & 1 & 1 & & 2 & & & & & & & & 4 \\
\hline Liatongus rhinoce & & & 4 & & & & & & & & & & & 4 \\
\hline Phana & & 1 & & & 1 & & & & & & & & & 2 \\
\hline Onthophagus mexicanus Bates, 1887 & & 1 & & & & & & & & & & 1 & 10 & 12 \\
\hline Cartwright, 1963 & & & & & & & & & & & & 4 & & 4 \\
\hline O. aureofuscus Bates, 1887 & 6 & 51 & 2 & 2 & 1 & & & & & & & & 3 & 65 \\
\hline O. pseudoundulans Zunino \& Halffter, 1988 & & & & & & & & & & & & & 1 & 1 \\
\hline O. chevrolati retusus Harold, 1869 & 349 & 1233 & 472 & 142 & 262 & 796 & 63 & 77 & 51 & 26 & 30 & 53 & 96 & 2933 \\
\hline Onthophagus sp. 1 & 1 & & & & & & & & & & & & & 1 \\
\hline Onthophagus s & & 1 & & & & & & & & & & & & 1 \\
\hline Onthophagus sp. 3 & & & & & 1 & & & & & & & & 1 & 2 \\
\hline Canthon humectus sayi Robinson, 1948 & & & 3 & & 1 & & & & & & & 19 & 11 & 34 \\
\hline Deltochilum mexi & 3 & 35 & 64 & 25 & 11 & 1 & & 1 & & & 2 & 14 & 29 & 185 \\
\hline Proculejus brevi & 1 & & 1 & 6 & & & & & 7 & & 3 & 4 & 3 & 25 \\
\hline Vindex agnoscendus $\mathrm{F}$ & 15 & 14 & 9 & 8 & 2 & & & 1 & 10 & 10 & 3 & 9 & 2 & 83 \\
\hline Undulifer incisus Truqui, 1857 & 15 & 17 & 11 & 7 & 2 & & & & 1 & & & 1 & & 54 \\
\hline & 391 & 135 & 570 & 193 & 286 & & 65 & 81 & 69 & 37 & 40 & 149 & 177 & 73512 \\
\hline
\end{tabular}

Hoplia Illiger, 1803. En el sitio denominado Puente Cervantes fue posible colectar una hembra de éste género atrapada en un charco de agua, la cuál consideramos que puede representar una especie no descrita.

Plusiotis Burmeister, 1844. En la trampa de luz de El Vivero se colectaron 16 machos y 3 hembras de $P$. adelaida (Cuadro 2). También se obtuvieron un macho y cuatro hembras de una especie de Plusiotis en proceso de descripción, que solo se ha localizado en las vertientes internas de las montañas situadas al norte de la ciudad de Oaxaca (comunicación personal de M. A. Morón).

Chrysina Kirby, 1827. Se colectaron 9 machos y 10 hembras de C. macropus atraídos por las luces entre las 20:30 y 23:00 hrs (Cuadro 2).

Rutelisca Bates, 1888. Bajo el alumbrado público de Santiago Xiacui se obtuvo un macho de $R$. flohri (Fig. 2). Es una especie subcortícola poco común registrada en bosques de coníferas y encinos de los estados de Guerrero, México, Oaxaca y el Distrito Federal (Jameson 2000). 


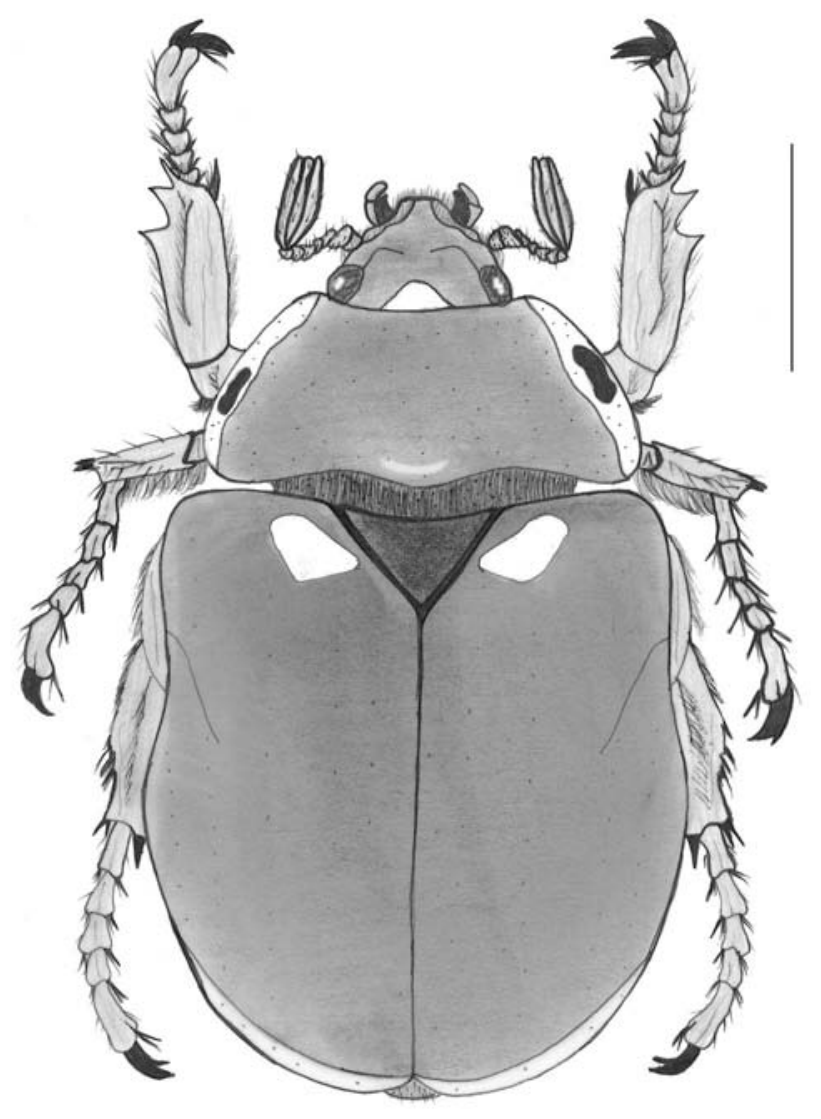

Figura 2. Rutelisca flohri Bates. Escala $10 \mathrm{~mm}$.

Paranomala Casey, 1915. De acuerdo con Ramírez-Ponce y Morón (2009) las especies americanas hasta ahora incluídas en el género Anomala Samouelle, no pertenecen al mismo clado que las especies eurasiáticas, por lo cuál, con pocas excepciones, se han reunido bajo el nombre de Paranomala Casey. En la región de Xiacui se obtuvieron representantes de nueve especies, todas capturadas al ser atraídas por la luz. Las especies mas abundantes fueron $P$. inconstans y $P$. undulata (Cuadro 2).

Dynastes Kirby, 1825. Solo se colectó un macho de D. hyllus bajo el alumbrado público de Santiago Xiacui. Es una especie poco abundante con amplia distribución en México asociada con ambientes muy diversos donde existan árboles de aguacate, ubicados entre los 500 y los 2,100 m de altitud. 
Golofa Hope, 1837. En la trampa de luz de El Vivero, y bajo el alumbrado público de San Pedro Nolasco y Santiago Xiacui se colectaron cuatro hembras y un macho de G. imperialis. En nuestra opinión, la sinonimía de G. imperialis con G. pizarro (Hope) sugerida por Ratcliffe y Cave (2006) es inadecuada, porque esta basada en el estudio de una muestra poco representativa de la distribución geográfica y ecológica de ambas especies, cuya amplia variación morfométrica fue analizada por Morón (1995).

Ancognatha Erichson, 1847. Se colectaron cuatro hembras de A. falsa atraídas por las luces y un macho de $A$. quadripunctata en un contenedor de agua. Es la primera vez que $A$. falsa y $A$. quadripunctata se encuentran en la misma localidad.

Xyloryctes Hope, 1837. Se estudiaron ocho hembras y dos machos de X. telephus atraídos por la trampa de luz en El Vivero, o colectados muertos a lo largo de los caminos hacia El Quelite, Bosque Madrigueras y El Vivero. Solo se colectó una muestra de esta especie que es muy abundante en la región. Entre el 21 y 23 de julio de 2003 en la trampa de luz de El Vivero fueron atraídos aproximadamente 800 ejemplares cada noche durante los primeros $5 \mathrm{~min}$ del crepúsculo. A lo largo del año siguieron presentándose en gran número, aunque disminuyeron paulatinamente hacia diciembre.

Heterogomphus Burmeister, 1847. Bajo el alumbrado público de Santiago Xiacui se obtuvo un macho de $H$. chevrolati de $36 \mathrm{~mm}$ de longitud.

Orizabus Farmairei, 1878. Se colectaron dos parejas de O. clunalis atraídas por las luces o sobre el camino al aserradero. Otra hembra obtenida bajo el alumbrado público de San Pedro Nolasco muestra caracteres similares a $O$. batesi, pero es necesario estudiar los machos para confirmar su identidad.

Ligyrus Burmeister, 1847. Aunque el cambio nomenclatorial propuesto por Ratcliffe (2002) para utilizar Tomarus Erichson en vez de Ligyrus Burmeister esta fundamentado en una ligera precedencia en fecha de publicación (enero 1847febrero 1847), es innecesario y provoca confusión en el uso del nombre Ligyrus, aplicado sin restricciones en publicaciones sucesivas durante casi 150 años, a la vez que Tomarus se ha empleado como nombre subgenérico subordinzado a Ligyrus en los últimos 35 años. La conservación del nombre genérico Ligyrus también ha sido apoyada por Escalona y Joly (2006). Bajo el alumbrado público de Santiago Xiacui se capturó un macho de L. (Ligyrodes) sallei.

Euphoria Burmeister, 1842. En forma directa se colectaron tres machos y una hembra de E. westermanni. Es una especie común, pero difícil de colectar con trampas cebadas, que habita en bosques de encino-pino situados entre los 1,400 y 2,000 m de altitud (Morón et al. 1997).

Cotinis Burmeister, 1842. Solo se colectó un macho de C. (Cotinis) mutabilis var. perbosci en un jardín de Santiago Xiacui. Es una especie poco común en los bosques de pinos y encinos. 
Peltotrichius Howden, 1968. En un charco de agua ubicado en el sitio Puente Cervantes se colectó un macho de $P$. linea (Fig. 3). Es una especie muy rara hasta ahora solo conocida por unos cuantos ejemplares colectados en los bosques húmedos de montaña de Molango, Hidalgo y Xalapa, Veracruz, situados entre los 1,400 y 1,650 m de altitud (Morón et al. 1997). El presente registro amplía notablemente su distribución hacia el sureste de México.

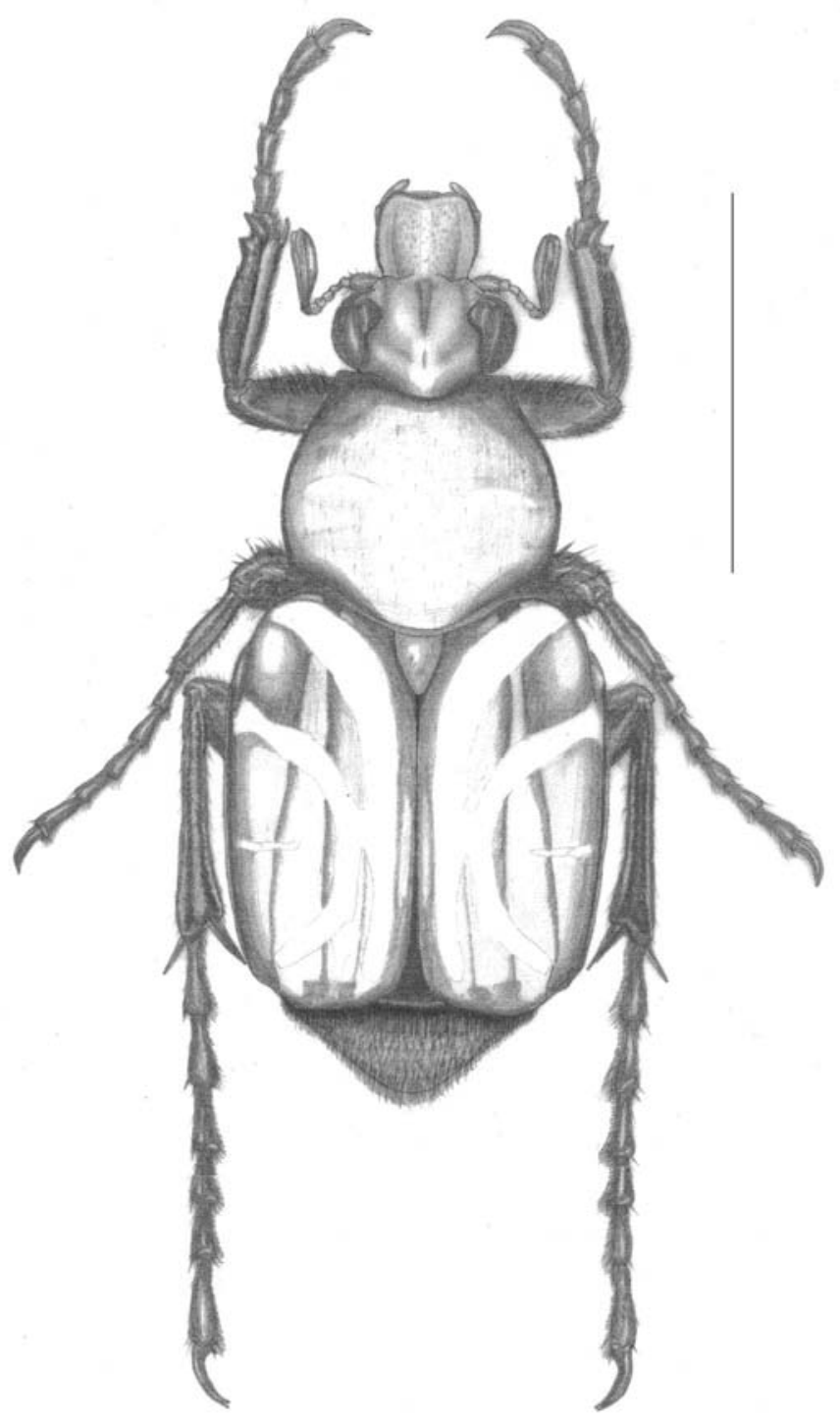

Figura 3. Peltotrichius linea Howden. Escala $10 \mathrm{~mm}$. 


\section{FAMILIA SCARABAEIDAE}

Canthon Hoffmannsegg, 1817. Fueron colectados 34 ejemplares de C. (Canthon) humectus sayi, 22 de ellos en coprotrampas, 10 en trampas NTP-80 y dos en excremento equino, a lo largo del camino a El Vivero (Cuadro 3). Esta subespecie es común en ambientes templados y húmedos del centro y sureste de México (Halffter 2003).

Deltochilum Eschscholtz, 1822. Se obtuvieron un total de 185 ejemplares de $D$. mexicanum, 66 de ellos capturados en coprotrampas y 119 en trampas NTP-80 (Cuadro 3). Esta especie tiene una amplia distribución desde México hasta Bolivia (Halffter 2003).

Copris Geoffroy, 1762. Se colectaron tres ejemplares de C. armatus en coprotrampas. Otros dos individuos (macho y hembra) se encontraron en estiércol equino y vacuno. Su hallazgo en la región de Santiago Xiacui amplía su distribución desde las montañas de Guerrero hacia el norte de Oaxaca.

Dichotomius Hope, 1838. Se capturaron cuatro ejemplares de D. colonicus, atraídos por las luces o bajo estiércol equino en el camino al El Aserradero. Es una especie coprófaga nocturna común en terrenos abiertos situados desde el nivel del mar hasta los 2,500 m de altitud (Kohlmann 2003).

Liatongus Reitter, 1893. En las cercanías de El Aserradero se obtuvieron cuatro machos de L. rhinocerulus (Fig. 4) en una trampa NTP-80. Se ha registrado en los estados de Durango, Guerrero, Jalisco, México, Morelos, Michoacán, Oaxaca, Sinaloa y Sonora (Navarrete-Heredia 2001).

Phanaeus MacLeay, 1819. Se colectaron dos hembras de Phanaeus (Phanaeus) damocles, una se encontró muerta sobre el camino al aserradero y la otra bajo el alumbrado público de Santiago Xiacui. Es una especie coprófaga que habita en bosques y praderas situadas entre los 1,800 y 2,900 m de altitud (Edmonds 2003).

Onthophagus Latreille, 1802. Se obtuvieron muestras de ocho especies pero solo fue posible identificar con precisión a cinco de ellas. La mayor parte de los ejemplares fueron capturados en coprotrampas y trampas NTP-80 y solo $O$. chevrolati retusus se encontró durante todo el año (Cuadro 3). Onthophagus aureofuscus únicamente se conoce de algunas localidades montañosas ubicadas en los estados de Durango, Oaxaca y Tlaxcala; y $O$. pseudoundulans solo se ha registrado de Guerrero y Oaxaca (Zunino y Halffter 1988). Solo cinco de los 2,933 ejemplares de $O$. chevrolati retusus no fueron colectados con trampas cebadas, una hembra se encontró dentro de un hongo podrido (Boletus sp.) en El Vivero, un macho se atrapó con una red de golpeo en el mismo sitio, dos machos fueron localizados en excremento de zorra en el sitio conocido como Bosque Madriguera, y una hembra fue hallada dentro de excremento vacuno en la Brecha Arrastradero. 


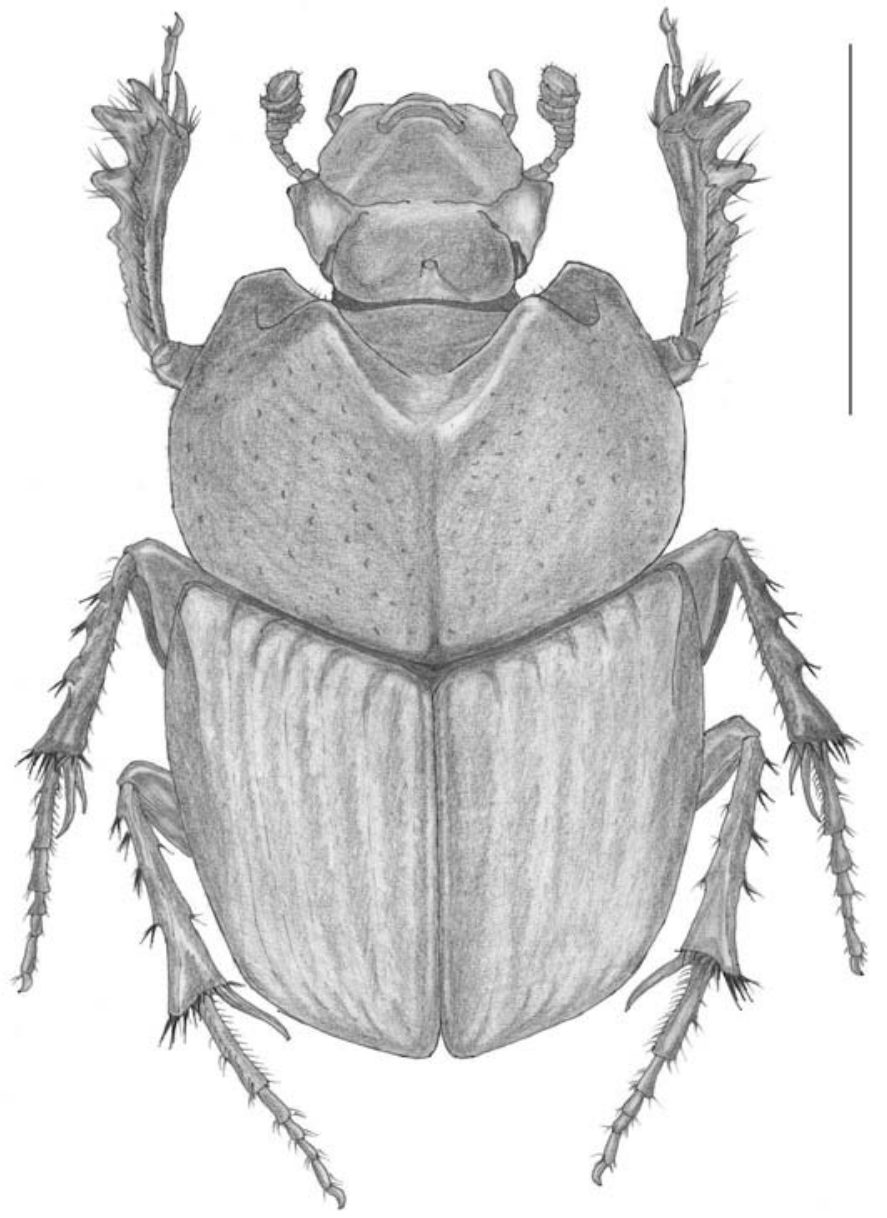

Figura 4. Liatongus rhinocerulus (Bates). Escala $10 \mathrm{~mm}$.

Labarrus Mulsant \& Rey, 1870. Se obtuvieron dos ejemplares de L. pseudolividus atraídos por las luces, y otros cinco en excremento vacuno. Es una especie con amplia distribución americana, la cuál fue identificada durante muchos años como Aphodius (Labarrus) lividus (Olivier, 1789), nombre que en realidad corresponde a un taxón introducido de Europa en algunas regiones del continente.

Haroldiellus Gordon \& Skelley, 2007. Se colectaron ocho ejemplares de H. sallei atraídos por las luces y otros cuatro se obtuvieron en excremento equino en el camino al aserradero.

Gonaphodiellus Schmidt, 1913. Se capturaron 46 ejemplares de una especie cercana a G. opisthius (Cuadro 3), tres de ellos en trampas NTP-80 y los demás en coprotrampas situadas a lo largo del camino al aserradero. 
Liothorax Motschulsky, 1859. En el sitio conocido como Río Cabañas en excremento equino se obtuvieron cuatro ejemplares de una especie cercana a $L$. innexus.

Ataenius Harold, 1867. Se encontraron cuatro ejemplares de una especie cercana a A. cribrithorax bajo el alumbrado público de Santiago Xiacui, y otro se localizó dentro de excremento equino en el camino al aserradero. Ataenius cribrithorax tiene una amplia distribución en México y en Las Antillas (Deloya 2003).

Geotrupes Latreille, 1796. Se colectaron ocho ejemplares de G. (C.) nebularum, seis de ellos en trampas NTP-80 ubicadas a los largo del camino al aserradero, una hembra en un charco de agua en El Quelite, y otra hembra muerta en el camino al sitio Río Cabañas. También se obtuvieron 14 ejemplares de $G$. (C.) sallei (ocho en coprotrampas, cuatro en NTP-80 y dos en colecta directa) en el camino hacia El Vivero (Cuadro 3).

Ochodaeus Lepeletier \& Serville, 1828. Se capturó una hembra de $O$. sp. aff. luridus atraída por la trampa de luz fluorescente instalada en El Vivero. Ochodaeus luridus se ha citado en ambientes de montaña con bosques de pino y encino en Guerrero y Durango (Bates 1889, Howden com.pers. 1999).

\section{FAMILIA PASSALIDAE}

Proculejus Kaup, 1868. Se colectaron 25 ejemplares de P. brevis (Cuadro 3), sobre todo dentro de troncos podridos de pinos y encinos a lo largo del camino al Aserradero. Es una especie de hábitos albodurámicolas que se han registrado en bosques mesófilos de montaña, mixtos de pino-encino, de encino húmedo y de pino situados entre los 1,300 a 3,000 m de altitud (Reyes-Castillo 2003).

Vindex Kaup, 1871. Durante todo el año fue posible colectar 83 ejemplares de $V$. agnoscendus dentro y debajo de troncos podridos de Pinus sp. (38), Quercus sp. (19) y Arbutus sp. (2) situados a lo largo del camino al Aserradero, entre los 2,000 y 3,240 $\mathrm{m}$ de altitud; caminando por el sotobosque durante mayo y julio (11); al vuelo entre las 11:30 y 12:00 hrs en el mes de mayo en El Quelite (3); en charcos de agua en Llano Verde (2); y bajo el alumbrado público de Santiago Xiacui (1) (Cuadro 3). Es una especie de hábitos subcortícolas, que algunas veces penetra en el aboduramen de Fagus, Pinus y Quercus (Reyes-Castillo 2003).

Undulifer Kaup, 1869. La muestra está formada por 54 ejemplares de $U$. incisus (Cuadro 3) de los cuales 44 se colectaron dentro y debajo de troncos podridos de Pinus sp. (33), Arbutus sp. (2), y Quercus sp. (9), cinco se colectaron en el piso del bosque (junio, agosto y septiembre), uno bajo el alumbrado público en Río Cabañas y cuatro con una trampa de luz en el vivero. Tiene hábitos albodurámicolas y se ha registrado en madera de encinos y pinos (Reyes-Castillo 2003). 


\section{FENOLOGÍA Y DIVERSIDAD}

La fenología de los escarabajos en la zona de estudio tiene una época bien definida en la cual la abundancia y diversidad de adultos es muy notable. La primera cresta de la gráfica coincide con la época de lluvias de 2003 y la segunda con el inicio de las precipitaciones de 2004 (Fig. 5). El notable incremento de la primera cresta se debe a una gran abundancia de individuos de una sola especie de Scarabaeidae (Onthophagus chevrolati retusus), la cuál representa el $67.7 \%$ de la colecta total y que en el periodo de junio-septiembre concentró al $81.92 \%$ del total de la muestra, el otro $18.07 \%$ se repartió entre los siguientes nueve meses. En esta época la abundancia de las otras familias se mantuvo discreta. En la segunda cresta la familia Scarabaeidae fue moderadamente abundante (pero mucho más diversa que en el inicio) respecto a lo mostrado por Melolonthidae pues en los meses de abril a junio se presentó su mayor abundancia y diversidad, representando el $66.26 \%$ del total colectado, mientras que Passalidae (2.3\%) y Scarabaeidae (31.42\%) sumaron el 33.73\% .

Los Melolonthidae mostraron una moderada abundancia y diversidad durante los primeros 5 meses (junio-octubre 2003) donde se registraron 20 entidades específicas y 81 ejemplares; para los siguientes cinco meses (noviembre 2003-marzo 2004) la actividad de las formas adultas fue casi nula pues solo 15 individuos de seis especies fueron colectados (Cuadro 2). Antes de iniciarse el periodo de lluvias de 2004 la actividad de Melolonthidae se incrementó radicalmente, tan solo en los meses de abril a junio se registraron 719 ejemplares de 43 especies $(87.75 \%$ del total de especies), de las cuales 27 especies no habían sido colectadas anteriormente (55.1 $\%$ ), además la muestra total de ejemplares colectados en estas fechas suman el 89.31 $\%$ del total de registros de la familia a lo largo del año. El incremento al final de la gráfica fue debido a una gran abundancia de individuos de cinco especies: Phyllophaga vetula (152), P. ravida (80), Diplotaxis sp. aff. jacala (60), Paranomala inconstans (129) y P. undulata (68), las cuales representan en tan solo tres meses el $60.74 \%$ del total de ejemplares colectados a lo largo del año (Fig. 5). Considerando la diversidad específica mensual es posible apreciar una época entre diciembremarzo donde la variedad de especies es mínima, coincidiendo con el periodo de estiaje (Fig. 5). Esta disminución notable es compartida con los Scarabaeidae.

Los Cetoniinae están pobremente representados en las muestras, a pesar de que se instalaron trampas con fruta fermentada y se revisaron las flores al alcance, solo se encontraron seis ejemplares de tres especies durante mayo y junio de 2004. Además de las dificultades para su colecta, la escasez del grupo es frecuente en los ambientes situados por arriba de los 2,000 m de altitud en la Zona de Transición Mexicana, aunque en tales sitios también se ha registrado la concentración de individuos en inflorescencias otoñales ruderales o en árboles frutales, pero esos hallazgos normalmente corresponden a especies euriecas de los géneros Euphoria y Cotinis (M.A. Morón obs. pers.). 


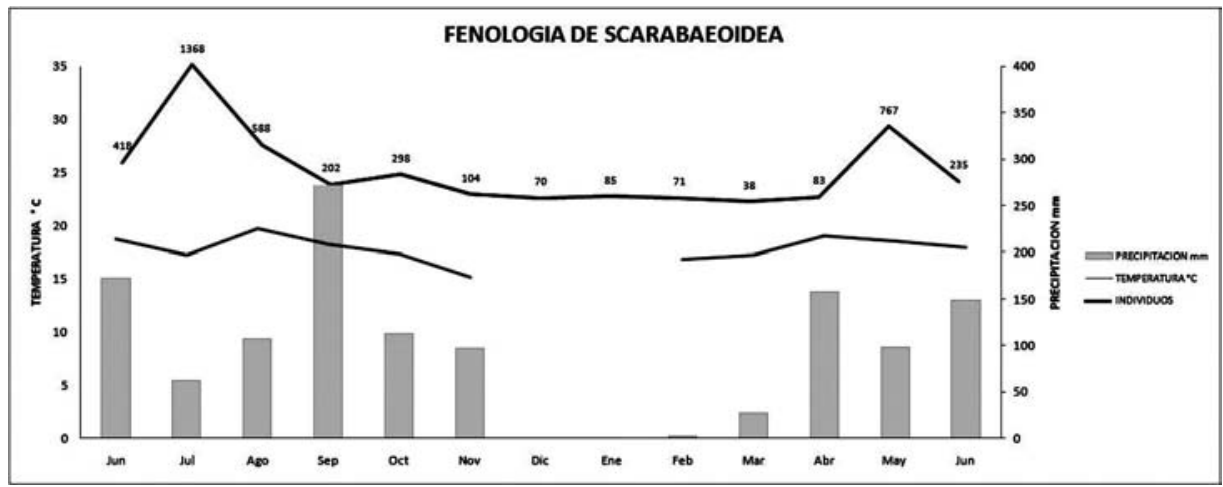

Figura 5. Fenología de la Superfamilia Scarabaeoidea en Santiago Xiacui, Oaxaca, 2003-2004.

Los Scarabaeidae mostraron una marcada actividad entre julio y octubre con una dominancia notable de Onthophagus chevrolati retusus que representa mas del $80 \%$ de la muestra total de la familia, la cual descendió hasta un $2 \%$ en marzo con respecto a su abundancia más alta registrada en julio (Fig. 3). Otras especies abundantes fueron Deltochilum mexicanum (185 ejemplares), Onthophagus aureofuscus (65), Gonaphodiellus aff. opisthius (46) y Canthon humectus (34) (Cuadro 3). En las NTP-80 se colectaron 1,657 ejemplares, y en las coprotrampas se obtuvieron 1,632 individuos, a pesar de que algunas de las trampas fueron saqueadas, robadas o inundadas. La localidad con mayor abundancia y diversidad para necrotrampas y coprotrampas fue El Arrastradero con 273 ejemplares en NTP-80 y 362 ejemplares en coprotrampas; en éste sitio también se registró la mayor diversidad de copronecrófagos, nueve especies, no obstante que fue el sitio con menor humedad. Los meses con mayor diversidad específica fueron agosto, octubre y mayo-junio durante los cuales se registraron ocho, diez y once especies respectivamente. La mayoría de las especies no registradas hasta antes del periodo más diverso (mayojunio) fueron colectadas dentro de excrementos de ganado vacuno o equino y solo dos especies con las trampas (Fig. 5). Los Geotrupidae están representados por dos especies de Geotrupes irregularmente obtenidas entre julio y diciembre de 2003 y de abril a junio de 2004 (Cuadro 3), lo cuál sugiere que los adultos no tienen actividad en el período invernal.

Los adultos de Passalidae son longevos y están activos durante todo el año, a diferencia de las otras familias de Scarabaeoidea, por lo que la presencia y número de ejemplares registrados a lo largo del año está fuertemente determinado por el esfuerzo de colecta en los restos xilosos. Los datos obtenidos para las tres especies 
de Proculejus, Vindex y Undulifer confirman lo anterior, aunque solo durante noviembre y diciembre no se registró alguna de ellas (Cuadro 3). Sin embargo su abundancia fue mayor cuando las lluvias se incrementaron, y disminuyeron entre octubre y enero cuando la humedad es menor (Fig. 5), a pesar de que la zona de estudio se caracteriza por la presencia de lluvias a lo largo de todo el año variando en intensidad dependiendo de la estación.

Representatividad del muestreo. En las curvas de acumulación de especies observadas (normal y aleatorizada) se comprobó que el muestreo aun no es representativo y que el inventario esta incompleto (Fig. 6). Las curvas de predicción de la riqueza de especies exponencial (Smax [1-e (-kx)]. Smax: 79.411. k: 0.18078. Pearsons Correlation sqred: 0.99433) e hiperbólica $(\operatorname{Smax} * x) /(B+x)$. Smax: 110.36. B: 6.7141. Pearsons Correlation sqred: 0.99801) estiman 79 y 110 especies respectivamente, es decir, el esfuerzo de colecta ha reunido durante 13 meses de muestreo el 90 y $65 \%$ de las especies estimadas por cada uno de los modelos. El modelo exponencial calcula que son necesarios 30 muestreos para colectar el $99 \%$ de las especies estimadas, mientras que la curva hiperbólica estima colectar solo 90 especies con el mismo esfuerzo de colecta $(81 \%$ del total de especies estimadas). Los estimadores no paramétricos predicen una riqueza específica de mas de 100 especies en la mayoría de los casos, el único estimador que predice una máxima riqueza de especies mucho menor a los anteriores al parecer es el menos preciso (Cuadro 4).

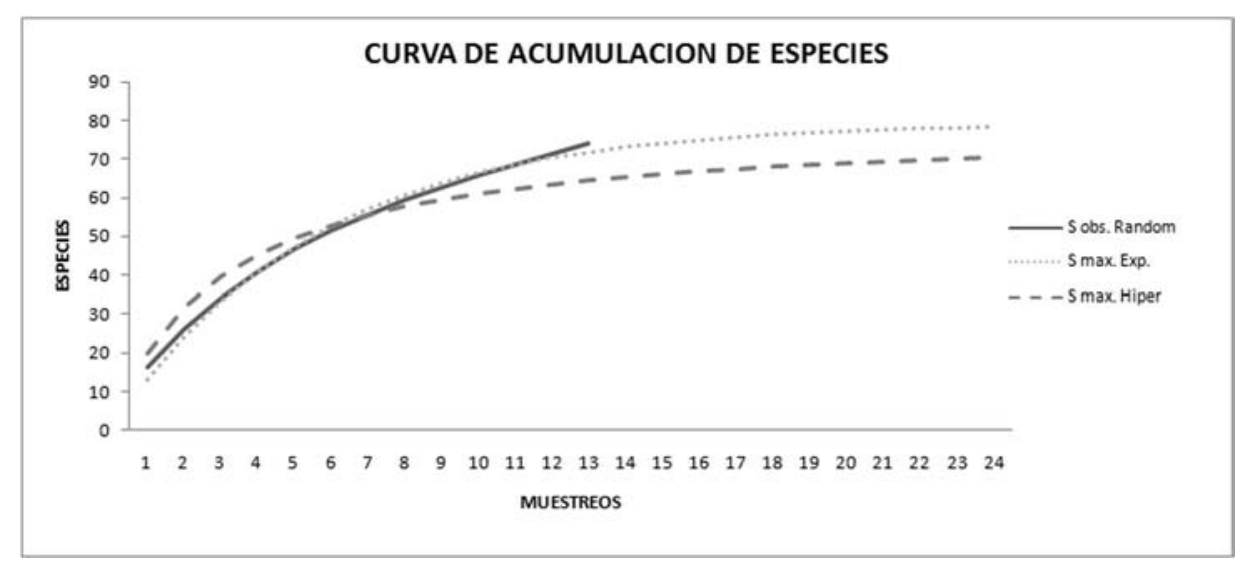

Figura 6. Curva de acumulación de especies en Santiago Xiacui, Oaxaca, 2003-2004. 
Cuadro 4. Estimadores no paramétricos de Smax.

\begin{tabular}{ccccc}
\hline SITIOS & Chao 2 & Jack 1 & Jack 2 & Bootstrap \\
\hline 1 & 302,96 & 19,12 & 0 & 19,12 \\
2 & 87,84 & 44,13 & 44,13 & 37,95 \\
3 & 83,13 & 57,84 & 65,39 & 48,13 \\
4 & 83,81 & 64,31 & 74,38 & 53,25 \\
5 & 83,88 & 72,11 & 83,94 & 59,74 \\
6 & 86,38 & 76,92 & 89,61 & 63,92 \\
7 & 92,01 & 82,73 & 96,65 & 68,83 \\
8 & 95,88 & 87,3 & 101,9 & 72,8 \\
9 & 99,16 & 91,1 & 106,27 & 76,14 \\
10 & 101,13 & 94,17 & 109,73 & 78,88 \\
11 & 104,56 & 98,14 & 114,14 & 82,39 \\
12 & 105,95 & 101,37 & 117,37 & 85,35 \\
13 & 107,14 & 103,54 & 119,76 & 87,17 \\
\hline
\end{tabular}

De las 74 especies colectadas durante la investigación 12 no se pudieron determinar específicamente debido a que se colectaron pocos ejemplares, no hay suficiente información sobre estos grupos, o bien, se trata de especies no descritas. De las 62 especies restantes cinco representan nuevos registros para el estado de Oaxaca: Phyllophaga lineatoides, P. eniba, Paranomala ayjikcala, Peltotrichius linea y Copris armatus.

\section{DISCUSIÓN}

Es claro que la constitución de la actual fauna de Santiago Xiacui en la Sierra Norte de Oaxaca es una combinación de elementos con distintas afinidades biogeográficas. Es parte de una área de transición entre las faunas propias de lugares más húmedos de la vertiente exterior y de zonas más secas en la vertiente interior, además de que la región esta ubicada en la confluencia de los grandes corredores biogeográficos de la Zona de Transición Mexicana (sensu Halffter 1976, 1978) que conectan los extremos meridionales de las Sierras Madre Oriental y del Sur. La información faunística obtenida durante este trabajo se esta complementando con otros datos para preparar un ensayo con enfoque zoogeográfico sobre la región.

Solo cuatro de las especies consideradas como plaga para otras zonas agrícolas de México (Morón 1999), se encontraron con cierta abundancia en Santiago Xiacui. Estas especies [Phyllophaga vetula (152 ejemplares), Paranomala inconstans (129), Phyllophaga ravida (80) y Macrodactylus mexicanus (23)] representan el 51.75\% del 
gremio filo-rizófago que a su vez constituye el $17.15 \%$ del total de las especies colectadas y en la zona pueden estar asociadas con cultivos de maíz, frijol, alfalfa, flores de ornato, frutales y hortalizas establecidos en la zona urbana y en la periferia. La ganadería no es una actividad económica importante en la comunidad, pues los habitantes solo cuentan con pocos animales principalmente para autoconsumo, transporte y para el arado. Es por ello que la mayor parte de las especies coprófagas colectadas son umbrófilas, destacando la abundancia de $O$. chevrolati retusus en la muestra total $(67.78 \%)$. También se registraron tres especies que prefieren bosques abiertos o áreas deforestadas que solo fueron colectadas cerca de la zona urbana, Onthophagus batesi (4), Dichotomius colonicus (4) y Copris armatus (5), cuya su baja densidad indica que aún no existen los espacios disponibles para su establecimiento.

Dentro del gremio sapro-rizo-xilófagos con importancia forestal destaca Xyloryctes telephus, que por su biomasa larvaria, su presencia a lo largo de todo el año y gran abundancia de adultos durante una época definida (junio-agosto) indica que es el elemento principal en la remoción de materia orgánica en el sotobosque, pues los otros dos géneros colectados de este gremio (Orizabus y Heterogomphus) presentaron una densidad poblacional muy baja.

Debido a que no se registraron especies filo-rizófagas con antecedentes dañinos lejos de la zona urbana, y a la escasa abundancia de especies coprófagas colonizadoras de ambientes alterados, podemos decir que el bosque se encuentra en buen estado de conservación. El bosque no presenta espacios abiertos o alteraciones antropogénicas significativas que pudieran permitir el establecimiento de estas especies, las cuales desplazarían a las especies nativas umbrófilas.

La mayor actividad de los escarabajos en el área de estudio coincide con la época de lluvias pues los Scarabaeidae mostraron un significativo aumento durante este periodo mientras que los Melolonthidae se mantuvieron discretos, aumentando su número y diversidad en el inicio y durante el mismo periodo. Consideramos que el esfuerzo de colecta en el gremio copro-necrófago está completo por lo que podemos afirmar que en la colecta total se tiene muy bien representadas esas especies. En el muestreo con trampa de luz es muy probable que se colecten especies adicionales a las registradas en este trabajo, pues las condiciones para el vuelo de estos organismos dependen en gran medida de circunstancias climáticas específicas desconocidas, y sería necesario incrementar el esfuerzo de colecta durante mas meses para aproximar el número de especies registradas con las predicciones de la estimación del número máximo de especies.

AGRADECIMIENTOS. Al Ing. Romeo Cid Flores y al M. en C. Rodolfo Alfredo Hernández Rea (Instituto Tecnológico Agropecuario de Oaxaca No. 23) por las sugerencias para mejorar una primera versión del manuscrito. A la comunidad de Santiago Xiacui por el apoyo y la hospitalidad durante los trabajos de campo. Esta publicación representa una contribución a la línea de investigación "Coleópteros Lamelicornios de América Latina" apoyado por la cuenta 2000910011 del Instituto de Ecología, A. C. 


\section{LITERATURA CITADA}

Arriaga, L., J.M. Espinoza, C. Aguilar, E. Martínez, L. Gómez \& E. Loa. 2000. Regiones terrestres prioritarias de México. Comisión Nacional para el Conocimiento y Uso de la Biodiversidad, México.

Bates, H.W. 1889. Pectinicornia and Lamellicornia. Biologia Centrali Americana, Zoología, Insecta Coleoptera vol. II, part 2. Supplement. Taylor and Francis, London.

Castillo, M.L. \& M.A. Morón. 1992. Observaciones sobre la degradación de madera por algunas especies de pasálidos (Coleoptera: Lamelicornia). Folia Entomológica Mexicana 84: 35-44.

Chao, A. 2004. Species richness estimation. Institute of Statistics National, Tsing Hua University.

Colwell, R.K. 2004. EstimateS: Statistical estimation of species richness and shared species from samples. Version 7.

Deloya, C. 2003. Subfamilia Aphodiinae. Pp. 75-93. In: Morón, M.A. (Ed.). Atlas de los escarabajos de México. Coleoptera : Lamellicornia, Volumen 2. Familias Scarabaeidae, Trogidae, Passalidae y Lucanidae. Argania editio, Barcelona.

Edmonds, D.W. 2003. Tribu Phanaeini. Pp. 58-65. In: Morón, M.A. (Ed.). Atlas de los escarabajos de México. Coleoptera : Lamellicornia, Volumen 2. Familias Scarabaeidae, Trogidae, Passalidae y Lucanidae. Argania editio, Barcelona.

Endrödi, S. 1966. Monographie der Dynastinae. (Col. Lam.). I Teil. Entomologische Abhandlungen Museum Tierkunde Dresden, 33: 1-457.

Escalona, H.E. \& L. J. Joly. 2006. El género Ligyrus Burmeister, 1847 en Venezuela (Coleoptera: Scarabaeidae: Dynastinae: Pentodontini). Boletín Sociedad Entomológica Aragonesa, no. 39: 111137.

Espinosa, E.T. 2003. ¿Cuántas especies hay? Los estimadores no paramétricos del Chao. Elementos, 2003: 53-56.

Espinosa, D., J.J. Morrone, C.Z. Aguilar \& J. Llorente. 2000. Regionalización Biogeográfica de México: Provincias Bióticas. Pp. 80-92. In: Llorente, J., E. González-Soriano \& N. Papavero (Eds.). Biodiversidad, taxonomia y biogeografía de artrópodos de México: Hacia una síntesis de su conocimiento. Vol. II. UNAM, México.

Favila, M.E. \& G. Halffter. 1997. The use of indicator groups for measuring biodiversity as related to community structure and function. Acta Zoológica Mexicana (n.s.) 72: 1-25.

Halffter, G. 1976. Distribución de los insectos en la zona de transición mexicana. Relaciones con la entomofauna de Norteamérica. Folia Entomológica Mexicana 35: 1-64.

Halffter, G. 1978. Un nuevo patrón de dispersión en la Zona de Transición Mexicana: el mesoamericano de montaña. Folia Entomológica Mexicana 39-40: 219-222.

Halffter, G. 2003. Tribu Scarabaeini. Pp. 21-43. In: Morón, M.A. (Ed.). Atlas de los escarabajos de México. Coleoptera : Lamellicornia, Volumen 2. Familias Scarabaeidae, Trogidae, Passalidae y Lucanidae. Argania editio, Barcelona.

Halffter, G., M.E. Favila \& V. Halffter. 1992. A comparative study of the structure of the scarab guild in Mexican tropical rain forest and derived ecosystems. Folia Entomológica Mexicana 84: 131-156.

INEGI. 1988. Anuario estadístico del estado de Oaxaca. INEGI, México.

Jameson, M. L. 2000. Synopsis of the Mexican and Guatemalan genera Rutelisca Bates and Metapachylus Bates (Coleoptera: Scarabaeidae: Rutelinae) with comments on classification of the subtribe Rutelina. Proceedings of the Entomological Society of Washington 102: 332-349.

Kohlmann, B. 2003. Tribu Coprini. Pp. 45-58. In: Morón, M.A. (Ed.). Atlas de los escarabajos de México. Coleoptera : Lamellicornia, Volumen 2. Familias Scarabaeidae, Trogidae, Passalidae y Lucanidae. Argania editio, Barcelona. 
Luis-Martínez, M.A., I. Vargas-Fernández \& J. E. Llorente. 1991. Lepidopterofauna de Oaxaca I: Distribución y fenología de los Papilionoidea de la Sierra de Juárez. Publicaciones especiales del Museo de Zoología, No. 3, Facultad de Ciencias, UNAM, México.

Moreno, C.E. 2001. Métodos para medir la biodiversidad. Manuales y Tesis, Sociedad Entomológica Aragonesa, vol. 1.

Moreno, C.E. \& G. Halffter. 2000. Assessing the completeness of bat biodiversity inventories using species accumulation curves. Journal Applied Ecology 37: 149-158.

Morón, M.A. 1985. Los insectos degradadores, un factor poco estudiado en los bosques de México. Folia Entomológica Mexicana 65: 131-137.

Morón, M.A. 1995. Review of the Mexican species of Golofa Hope (Coleoptera: Melolonthidae, Dynastidae). Coleopterists Bulletin 49 (4): 343-386.

Morón, M.A. 1997. Inventarios faunísticos de los Coleoptera Melolonthidae Neotropicales con potencial como bioindicadores. Giornale italiano Entomologia 8: 265-274.

Morón, M.A. 1999. Coleoptera Melolonthidae. Pp.43-59. In: Deloya, C. \& J. Valenzuela (Eds.) Catálogo de insectos y ácaros plaga de los cultivos agrícolas de México. Publicaciones especiales No. 1. Sociedad Mexicana de Entomología, A. C. México.

Morón, M.A. 2001. Larvas de escarabajos del suelo en México (Coleoptera: Melolonthidae). Acta Zoológica Mexicana (n.s.) Número especial 1: 111-130.

Morón, M.A. (Ed.). 2003 a. Atlas de los escarabajos de México. Coleoptera : Lamellicornia, Volumen 2. Familias Scarabaeidae, Trogidae, Passalidae y Lucanidae. Argania editio, Barcelona.

Morón, M.A. 2003 b. Diversidad, distribución e importancia de las especies de Phyllophaga Harris en México (Coleoptera : Melolonthidae). Pp. 1-27. In : Aragón, A., M.A.Morón \& A. Marín (Eds.). Estudios sobre coleópteros del suelo en América. Publicación especial de la Benemérita Universidad Autónoma de Puebla, México.

Morón, M.A. \& A. Aragón. 2003. Importancia ecológica de las especies americanas de Coleóptera Scarabaeoidea. Duguesiana 10(1): 13-29.

Morón, M.A., S. Hernández-Rodríguez \& A. Ramírez-Campos. 1996. El complejo "gallina ciega" (Coleoptera: Melolonthidae) asociado con la caña de azúcar en Nayarit, México. Folia Entomológica Mexicana 98: 1-44.

Morón, M.A., B.C. Ratcliffe \& C. Deloya. 1997. Atlas de los escarabajos de México. Volumen 1. Familia Melolonthidae. CONABIO y Sociedad Mexicana de Entomología, A. C. México.

Morón, M.A. \& R. Terrón, 1984. Distribución altitudinal y estacional de los inséctos necrófilos en la sierra Norte de Hidalgo, México. Acta Zoológica Mexicana 3: 1-47.

Navarrete-Heredia, J.L. 2001. New Mexican state records for Oniticellus rhinocerulus Bates and Euoniticellus intermedius (Reiche) (Scarabaeidae: Scarabaeinae). Coleopterists Bulletin 55(4): 500.

Ramírez-Ponce, A. \& M.A. Morón. 2009. Relaciones filogenéticas del género Anomala (Coleoptera: Melolonthidae: Rutelinae). Revista Mexicana de Biodiversidad 80: 1-37.

Ratcliffe, B.C. 2002. Dynastinae. Pp. 64-67. In: Arnett, R.H. Jr., M.C. Thomas, P.E. Skelley \& J.H. Frank (Eds.). American Beetles. Vol. 2. Polyphaga: Scarabaeoidea through Curculionoidea. CRC Press, Boca Raton.

Ratcliffe, B.C. \& R.D. Cave. 2004. The Dynastine scarab beetles of Honduras, Nicaragua and El Salvador (Coleoptera: Scarabaeidae: Dynastinae). Bulletin University of Nebraska State Museum 21:1-424.

Reyes-Castillo, P. 1970. Coleoptera Passalidae. Morfología y división en grandes grupos; géneros americanos. Folia Entomológica Mexicana 20-22: 1-240. 
Reyes-Castillo, P. 2003. Familia Passalidae. Pp. 135-168. In: Morón, M.A. (Ed.). Atlas de los escarabajos de México. Coleoptera : Lamellicornia, Volumen 2. Familias Scarabaeidae, Trogidae, Passalidae y Lucanidae. Argania editio, Barcelona.

Soberón, J. \& J. Llorente. 1993. The use of species accumulation functions for the prediction of species richness. Conservation Biology 7: 480-488.

Zunino, M. \& G. Halffter. 1988. Análisis taxonómico, ecológico y biogeográfico de un grupo americano de Onthophagus (Coleoptera: Scarabaeidae). Monografía IX. Museo Regionale di Scienze Naturali, Torino. 